\title{
The HIV care cascade for adolescents initiated on antiretroviral therapy in a health district of South Africa: a retrospective cohort study
}

\author{
Roxanna Haghighat ${ }^{{ }^{*}}$ (D) Elona Toska ${ }^{2,3}$, Nontuthuzelo Bungane ${ }^{4}$ and Lucie Cluver ${ }^{1,5}$
}

\begin{abstract}
Background: Little evidence exists to comprehensively estimate adolescent viral suppression after initiation on antiretroviral therapy in sub-Saharan Africa. This study examines adolescent progression along the HIV care cascade to viral suppression for adolescents initiated on antiretroviral therapy in South Africa.

Methods: All adolescents ever initiated on antiretroviral therapy $(n=1080)$ by 2015 in a health district of the Eastern Cape, South Africa, were interviewed in 2014-2015. Clinical records were extracted from 52 healthcare facilities through January 2018 (including records in multiple facilities). Mortality and loss to follow-up rates were corrected for transfers. Predictors of progression through the HIV care cascade were tested using sequential multivariable logistic regressions. Predicted probabilities for the effects of significant predictors were estimated by sex and mode of infection.

Results: Corrected mortality and loss to follow-up rates were 3.3 and 16.9\%, respectively. Among adolescents with clinical records, $92.3 \%$ had $\geq 1$ viral load, but only $51.1 \%$ of viral loads were from the past 12 months. Adolescents on ART for $\geq 2$ years (AOR 3.42 [95\% Cl 2.14-5.47], $p<0.001$ ) and who experienced decentralised care (AOR 1.39 [95\%Cl 1.06-1.83], $p=0.018$ ) were more likely to have a recent viral load. The average effect of decentralised care on recent viral load was greater for female (AOR $2.39[95 \% \mathrm{Cl} 1.29-4.43], p=0.006)$ and sexually infected adolescents (AOR $3.48[95 \% \mathrm{Cl} 1.04-11.65], p=0.043)$. Of the total cohort, $47.5 \%$ were recorded as fully virally suppressed at most recent test. Only $23.2 \%$ were recorded as fully virally suppressed within the past 12 months. Younger adolescents (AOR 1.39 [95\%Cl 1.06-1.82], $p=0.017$ ) and those on ART for $\geq 2$ years (AOR 1.70 [95\%Cl 1.12-2.58], $p=0.013$ ) were more likely to be fully viral suppressed.
\end{abstract}

Conclusions: Viral load recording and viral suppression rates remain low for ART-initiated adolescents in South Africa. Improved outcomes for this population require stronger engagement in care and viral load monitoring.

Keywords: Adolescent, HIV, Antiretroviral therapy, South Africa, Care cascade, Continuum of care

* Correspondence: roxanna.haghighat@spi.ox.ac.uk

'Department of Social Policy and Intervention, University of Oxford, 32 Wellington Square, Oxford OX1 3DW, UK

Full list of author information is available at the end of the article

C C The Author(s). 2021 Open Access This article is licensed under a Creative Commons Attribution 4.0 International License, which permits use, sharing, adaptation, distribution and reproduction in any medium or format, as long as you give appropriate credit to the original author(s) and the source, provide a link to the Creative Commons licence, and indicate if changes were made. The images or other third party material in this article are included in the article's Creative Commons licence, unless indicated otherwise in a credit line to the material. If material is not included in the article's Creative Commons licence and your intended use is not permitted by statutory regulation or exceeds the permitted use, you will need to obtain permission directly from the copyright holder. To view a copy of this licence, visit http://creativecommons.org/licenses/by/4.0/. The Creative Commons Public Domain Dedication waiver (http://creativecommons.org/publicdomain/zero/1.0/) applies to the data made available in this article, unless otherwise stated in a credit line to the data. 


\section{Background}

The expansion of access to antiretroviral therapy (ART) has enabled more children vertically infected with HIV to survive into adolescence [1]. In parallel, the high rate of new HIV infections through sexual transmission among youth has contributed to a growing population of adolescents living with HIV. Of the estimated 2.1 million adolescents living with HIV, 85\% reside in sub-Saharan Africa [2,3]. Concern about the growing population of adolescents requiring lifelong ART has drawn increasing global attention to the need to better monitor this key population and to tailor treatment services $[1,4]$.

Previous studies have demonstrated that, even on treatment, adolescents exhibit the worst health outcomes compared to all other age groups $[2,5]$. In South Africa, which houses the world's largest ART programme, adolescents have repeatedly demonstrated the lowest rates of retention in care and viral suppression compared to other age groups [6]. The HIV care cascade has been widely used among various patient populations to identify progress at critical stages along the continuum of care from HIV testing to ART initiation and viral suppression [7]. This tool allows for both the monitoring of patients' health and identification of key gaps in care that can be targeted by interventions in alignment with the UNAIDS 90-90-90 treatment target for $2020[8,9]$.

Although progress along the HIV care cascade has been well-documented globally for adults and adult key populations, less evidence exists for adolescents living with $\operatorname{HIV}[6,7,9]$. A recent retrospective analysis of individually linked records from South Africa's National Health Laboratory Service database estimated that 66\% of older adolescents (15-19 years old) had initiated ART by 2016 [10]. While the study provides a robust estimate for the second cascade step of ART initiation, it does not extend analyses to evaluate rates of viral suppression for the third target in the care cascade.

In South Africa, one systematic review of data from youth living with HIV estimated that only $10 \%$ were virally suppressed as of 2013, before implementation of universal test and treat in 2016 [6]. However, the review estimated viral suppression using rates reported for patients aged 929 , well beyond the window of adolescence [11]. Similarly, the 2017 South African National HIV Prevalence, Incidence, Behaviour, and Communication Survey estimated viral suppression for youths aged 15-24, with no discrete data for all adolescents aged 10-19 [12]. This lack of disaggregated data for adolescents limits the applicability of findings for this key population [4]. Furthermore, viral suppression in the survey was calculated by active collection and testing of blood samples, not evaluation of clinical records that would be available to healthcare providers. Research is urgently required to understand the current reality of adolescent HIV care progression to the final cascade step of viral suppression in South Africa, with data that reflects contexts beyond well-resourced clinics or urban centres [13].

In this study, we evaluate the progression of ARTinitiated adolescents to viral suppression along the HIV care cascade in South Africa, including operational outcomes as well as mortality and retention in care, using data up to 2018 from multiple healthcare facilities within South Africa's decentralised public healthcare system. In addition, we report associations between stages of the cascade and adolescents' sociodemographic and treatment-related characteristics.

\section{Methods}

\section{Participants and procedures}

In this longitudinal cohort study, data were analysed from clinical records and interviews with ARTinitiated adolescents in a health district of the Eastern Cape, South Africa. The study setting was specifically selected to reflect poverty and healthcare contexts similar to other low-income Southern African contexts and included rural, peri-urban, and urban locations [14]. The province is characterised by high HIV and TB burden, and significant challenges for this province's healthcare system include limited infrastructure and human resource challenges [15]. Adolescent participants were recruited from March 2014 through September 2015, as previously described [16]. All healthcare facilities within the health district that provided ART to at least five adolescents were included in the study $(n=52$, including eight hospital wards, five community health centres, and 39 primary care clinics). At each healthcare facility, patient registers were reviewed to identify all adolescents aged 10-19 years who had ever initiated ART, including those who had defaulted in care or been lost to follow up (LTFU). Adolescents identified through clinical records were traced to $>180$ communities, approached for study participation, and, if consenting, interviewed at their preferred location.

\section{Data collection \\ Clinical record review}

At each of the 52 healthcare facilities, routine clinical records were searched for every study participant, across both paper-based and electronic records. This intensive data collection approach enables the extraction of participants' records from all included facilities where they may have received care, including undocumented silent transfers to a new facility. Data were extracted in two rounds using a standardized form, covering records from 2014 to 2015 and 2016-2017 respectively, and included plasma viral load, CD4 cell count, and WHO staging. 


\section{Adolescent interviews}

At two study waves ( 0 and 18 months follow-up), adolescents completed tablet-based surveys in their preferred language, with the support of research assistants trained in working with South African adolescents. Surveys assessed adolescents' experiences at home, in their communities, and in healthcare settings, and questions were developed to be understandable and non-stigmatising through extensive stakeholder consultation, including with South African adolescents living with HIV [16, 17]. For the present analyses, self-reported data were only used to determine participants' sociodemographic and treatment-related characteristics. Further study information, including study protocol, is available at www.mzantsiwakho.org.za.

\section{Measures}

Operational outcomes were evaluated in the HIV care cascade between ART initiation and viral suppression and included the following: availability of any clinical records, having at least one viral load recorded within clinical records, and having a viral load recorded within the past 12 months (backdated from the date of extraction). Finally, viral suppression was measured at two thresholds (HIV-1 RNA $<1000$ copies/mL and full viral suppression designated at HIV-1 RNA $<50$ copies $/ \mathrm{mL}$ ) [18]. Viral suppression was determined using the most recent viral load available across records, including in multiple facilities. Mortality was determined using both clinical records and community tracing through May 2018. LTFU was defined as recorded in participants' most recent clinical records, adjusted for mortality and silent transfers into care at new facilities. Silent transfers were identified when a patient re-entered care in a new facility without an official notification to the former facility of care. Participants without any available clinical records were assumed to be LTFU.

In total, seven covariates were included in analyses [1]: Age (younger adolescents aged 10-14 years vs. older adolescents aged 15-19 years) [2]; sex [3]; residential location at baseline (urban vs. rural) [4]; sexual vs. vertical mode of infection (determined following existing subSaharan African paediatric cohorts: age of ART initiation cut-off [ $\leq 10$ years] [19], validated and updated with a detailed algorithm that considered other strong evidence (i.e. self-reported sexual history and parental death) [20] [5]; time on ART ( $\geq 2$ vs. $<2$ years, measured from ART initiation to date of viral load) [6]; experiencing decentralised care (either exclusively at primary care clinics or experienced down-referral from a higher- to lower-level facility); and [7] mortality. For analyses of viral suppression, age at most recent viral load was binarized and used as the age covariate (10-14 vs. 15-19 years). For other outcomes, binarized age at study enrolment was used (10-14 vs. 15-19 years). Mortality was included as a covariate only for analyses of operational outcomes (having a clinical record and having at least one viral load in clinical records).

\section{Statistical analysis}

First, we summarised sociodemographic and treatmentrelated characteristics of the cohort using descriptive statistics. Second, we calculated the relevant proportion of participants who had reached each step of the HIV care cascade, including operational outcomes. The proportion of participants with clinical records was measured out of the total study cohort, and the proportion of participants with available viral loads was calculated relative to participants with available clinical records. The proportions of participants with viral loads recorded in the past 12 months and viral suppression were calculated relative to the number of participants with at least one recorded viral load.

Third, we tested associations between each step of the HIV care cascade and socio-demographic and treatmentrelated predictors, using a sequential multivariable logistic regression approach following Hosmer and Lemeshow [21]. All covariates were simultaneously included in the first model and then removed sequentially: the second and third models retained only variables significant at $p<0.10$ and $p<0.05$, respectively. For each cascade outcome, the final model controlled for test multiplicity, using the Benjamini-Hochberg step-up procedure to correct for false discovery rate [22]. For all models, correlation matrices indicated no multicollinearity between variables. All possible two- and three-way interactive effects between covariates significant in the final model were also tested and corrected using the Benjamini-Hochberg procedure and the Wald test for significance. Fourth, predicted probabilities for covariates significant in the final model were estimated by sex and mode of infection [23]. Multivariable logistic regressions and marginal probability models were performed using SPSS version 23 (IBM Corp, Armonk, NY) and STATA/SE 15.1 (StataCorp, College Station, $\mathrm{TX})$, respectively.

\section{Ethical approval}

Ethical approval for this study was given by the University of Oxford (SSD/CUREC2/12-21), University of Cape Town (CSSR 2013/4; 2017/3), Eastern Cape Departments of Health and Basic Education, and ethical review boards of all participating healthcare facilities. At both study waves, adolescent participants and their caregivers provided voluntary, informed, and written consent for participation, including interviews and access to adolescents' clinical records. Initial review of patient registers in healthcare facilities was only used to identify eligible participants, and further access to clinical records was 
obtained only after adolescents consented to study participation. In cases of low literacy among adolescents or caregivers, all information and consent procedures were read aloud in the participant's preferred language. There were no financial incentives for study participation, but all participants received a certificate of participation, snacks, and a small gift pack including pencils and soap. Adolescents who refused to participate were still given snacks. To minimise risk of stigma, all potentially publicly available materials, such as participation certificates and general information pamphlets, referred to the study as one about general adolescent health in South Africa.

\section{Results}

A total of 1080 ART-initiated adolescents were recruited into the study. Years of ART initiation ranged from 2000 to 2017, providing 6921 person-years of study follow-up across participants. Median age at study enrolment was 13 years (IQR: 11-16 years), and $55.3 \%$ of participants were female (Table 1 ). Most participants were living in urban locations (76.7\%) and vertically infected (74.8\%). In the total cohort, mortality and corrected LTFU rates (including those without clinical records) were $3.3 \%(n=36)$ and $16.9 \%(n=183)$, respectively. Overall, $37.9 \%$ of all facility-designated LTFU patients $(n=95)$ were found to be misclassified: $7.4 \%(n=7)$ due to unrecorded mortality, and 30.5\% $(n=29)$ from re-entry into care after silent transfer to a new facility.

Of the total adolescent cohort, $88.1 \%(n=951)$ had at least one clinical record available (Fig. 1). Attending multiple care facilities was common, with $29.8 \%(n=283)$ of participants having records from at least two facilities. In total, 51.3\% $(n=488)$ experienced decentralised care.

Among those with clinical records, median age at ART initiation was 9 years (IQR: 6-12 years), and median follow-up time since ART initiation was 7.2 years (IQR: 4.7-9.8 years). At baseline on ART, the majority of adolescents were viraemic, with $72.1 \%$ reporting detectable viral loads ( $\geq 50$ copies $/ \mathrm{mL}$ ) (Table 1$)$. At baseline, at least $19.0 \%$ were severely immunocompromised (CD4 <200 cells $/ \mathrm{mm}$ [3]), and at least $22.3 \%$ were either moderately or severely symptomatic (WHO Clinical Stage III/IV).

Among those with clinical records, a further 92.3\% $(n=878)$ had at least one viral load recorded within their files (Fig. 1). Of those with any viral load data, $75.4 \%(n=662)$ and $51.1 \%(n=449)$ had their most recent viral load recorded in the past 24 and 12 months, respectively. Median age at most recent viral load was 14 years (IQR: 12-17 years). Approximately equal proportions of adolescents had their most recent viral load recorded at a hospital $(39.0 \%, n=342)$ and a primary care clinic $(39.7 \%, n=349)$, with $21.3 \%$ $(n=187)$ recorded at a community health centre. At most recent viral load, $74.1 \%(n=651)$ were on firstline ART with an NNRTI backbone, and 19.2\% ( $n=$ $169)$ were on second-line ART with a PI backbone. A further $6.6 \%(n=58)$ were on an NRTI-only or unspecified ARV regimen.

Table 1 Baseline demographic and clinical characteristics of study cohort

\begin{tabular}{|c|c|}
\hline & $\begin{array}{l}\text { Number of participants, } \\
\%(n=1080)\end{array}$ \\
\hline \multicolumn{2}{|l|}{ Sex } \\
\hline Female & $597(55.3 \%)$ \\
\hline Male & $483(44.7 \%)$ \\
\hline \multicolumn{2}{|l|}{ Location } \\
\hline Urban & $828(76.7 \%)$ \\
\hline Rural & $252(23.3 \%)$ \\
\hline \multicolumn{2}{|l|}{ Age } \\
\hline Age at study enrolment (yrs, median (IQR)) & $13(11-16)$ \\
\hline Age at ART initiation (yrs, median $(\mathrm{IQR}))^{*}$ & $9(6-12)$ \\
\hline \multicolumn{2}{|l|}{ Mode of infection } \\
\hline Vertically infected $(n, \%)$ & $808(74.8 \%)$ \\
\hline Sexually infected $(n, \%)$ & $272(25.2 \%)$ \\
\hline \multicolumn{2}{|l|}{ Year of ART initiation } \\
\hline $2000-2009$ & $436(40.4 \%)$ \\
\hline 2010-2013 & $373(34.5 \%)$ \\
\hline 2014-2017 & $114(10.6 \%)$ \\
\hline Data not available & $157(14.5 \%)$ \\
\hline \multicolumn{2}{|l|}{ Baseline viral load } \\
\hline$<50$ copies $/ \mathrm{mL}$ & $99(9.2 \%)$ \\
\hline 51-999 copies/mL & $401(37.1 \%)$ \\
\hline$\geq 1000$ copies $/ \mathrm{mL}$ & $378(35.0 \%)$ \\
\hline Data not available & $202(18.7 \%)$ \\
\hline \multicolumn{2}{|l|}{ Baseline CD4 count } \\
\hline$<200$ cells $/ \mathrm{mm}^{3}$ & 205 (19.0\%) \\
\hline 200-349 cells $/ \mathrm{mm}^{3}$ & $192(17.8 \%)$ \\
\hline$\geq 350$ cells $/ \mathrm{mm}^{3}$ & $515(47.7 \%)$ \\
\hline Data not available & $169(15.6 \%)$ \\
\hline \multicolumn{2}{|l|}{ Baseline WHO Clinical Stage } \\
\hline Stage I & $283(26.2 \%)$ \\
\hline Stage ॥ & $167(15.5 \%)$ \\
\hline Stage III & $212(19.6 \%)$ \\
\hline Stage IV & $29(2.7 \%)$ \\
\hline Data not available & $388(35.9 \%)$ \\
\hline \multicolumn{2}{|l|}{ Follow-up time } \\
\hline $\begin{array}{l}\text { Study follow-up time since ART initiation } \\
(y r s \text {, median }(\mathrm{IQR}))^{*}\end{array}$ & $7.2(4.7-9.8)$ \\
\hline
\end{tabular}

ART: Antiretroviral therapy; IQR: Interquartile Range; LTFU: Lost to follow-up * Median values calculated among participants with available clinical records 


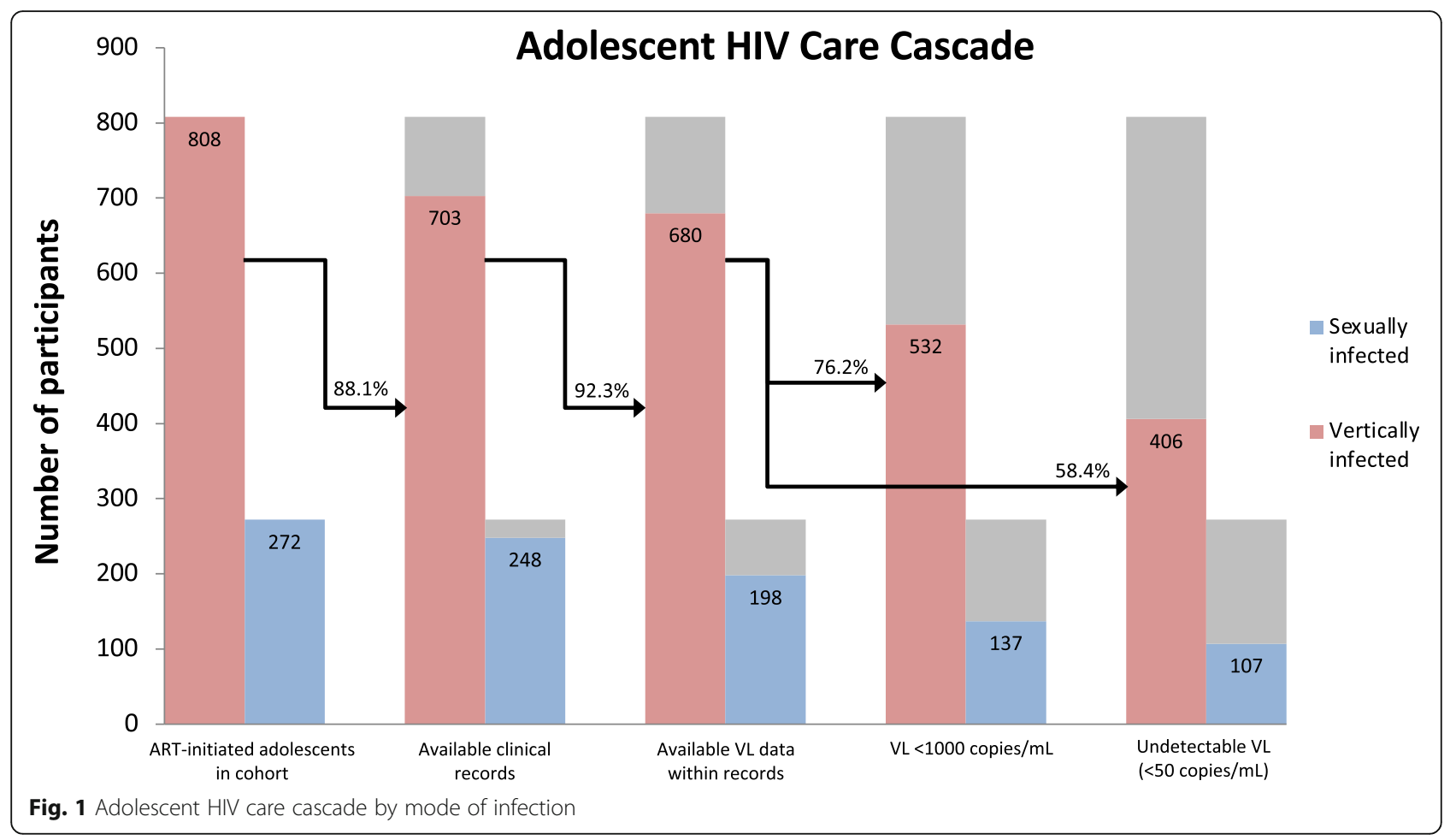

At most recent viral load, 76.2\% ( $n=669)$ of those with viral load data were virally suppressed at $<1000$ copies/ $\mathrm{mL}$, and $58.4 \%(n=513)$ were fully virally suppressed at $<$ 50 copies $/ \mathrm{mL}$ (Fig. 1). This rate corresponds to $47.5 \%$ full viral suppression at most recent viral load for the total adolescent cohort. Only $23.2 \%(n=251)$ of the total cohort demonstrated full viral suppression within the past 12 months.

In the final multivariable model, male (AOR 1.55 [95\%CI 1.05-2.28], $p=0.026)$ and sexually infected adolescents (AOR 1.71 [95\%CI 1.06-2.74], $p=0.028$ ) were more likely to have an available clinical record (Table 2). However, sexual infection (AOR 0.28 [95\%CI 0.15-0.52], $p<0.001$ ) and older age at study enrolment (AOR 0.32 [95\%CI 0.16-0.62], $p=0.001$ ) were associated with lower likelihood of having at least one recorded viral load. Male (AOR 2.36 [95\%CI 1.28-4.37], $p=0.006$ ) and rural-living adolescents (AOR 2.13 [95\%CI 1.07-4.27], $p=0.033$ ) were more likely to have a viral load available in clinical records (Table 2).

Longer time on ART (AOR 3.42 [95\%CI 2.145.47], $p<0.001)$ and experiencing decentralised care (AOR 1.39 [95\%CI 1.06-1.83], $p=0.018$ ) were associated with having a viral load recorded in the past 12 months (Table 2). Longer time on ART was also associated with viral suppression at $<1000$ copies $/ \mathrm{mL}$ (AOR 1.72 [95\%CI 1.09-2.72], $p=0.020$ ). However, adolescents who were older at most recent viral load (AOR 0.54 [95\%CI 0.39-0.75], $p<0.001$ ) and rural- residing (AOR 0.66 [95\%CI $0.46-0.93], \quad p=0.019$ ) were less likely to be virally suppressed at this level. Similarly, adolescents on ART for longer were more likely to be fully virally suppressed at $<50$ copies $/ \mathrm{mL}$ (AOR 1.70 [95\%CI 1.12-2.58], $p=0.013$ ), but adolescents who were older at most recent viral load were less likely to be fully virally suppressed (AOR 0.72 [95\%CI 0.55-0.94], $p=0.017$ ). Full results for all model stages for each cascade outcome are provided in Additional Files 1 and 2. No significant three-way interactions were found between variables significant in the final models for any outcome.

In the marginal probability model, the average effect of decentralised HIV care on likelihood of having a viral load in the past 12 months was greater for females than males (AOR 2.39 [95\%CI 1.29-4.43], Wald $\chi^{2}=7.60, p=$ 0.006 ), and greater for sexually infected adolescents than their vertically infected counterparts (AOR 3.48 [95\%CI 1.04-11.65], Wald $\chi^{2}=4.10, p=0.043$ ) (Fig. 2).

\section{Discussion}

This study provides a comprehensive estimate of the current reality of adolescent HIV care and progression to viral suppression through the HIV care cascade in South Africa's public healthcare system, including operational outcomes. These results highlight not only the low rate of past-year viral suppression among adolescents but also the importance of timely viral load monitoring. To our knowledge, this is the first study to 
Table 2 Final models for associations between HIV care cascade outcomes and socio-demographic and treatment-related characteristics

\begin{tabular}{|c|c|c|c|c|c|}
\hline & $\begin{array}{l}\text { Available clinical } \\
\text { record }(n=951) \\
\text { AOR }(95 \% \mathrm{Cl}) ; \\
p \text { value }\end{array}$ & $\begin{array}{l}\text { Available VL data } \\
\text { within records }(n=878) \\
\text { AOR }(95 \% \mathrm{Cl}) ; p \text { value }\end{array}$ & $\begin{array}{l}\text { VL recorded in past } \\
12 \text { months }(n=449) \\
\text { AOR }(95 \% \mathrm{Cl}) ; p \text { value }\end{array}$ & $\begin{array}{l}\text { Most recent } \mathrm{VL}<1000 \\
\text { copies } / \mathrm{mL}(n=669) \\
\text { AOR }(95 \% \mathrm{Cl}) ; p \text { value }\end{array}$ & $\begin{array}{l}\text { Most recent } \mathrm{VL}<50 \\
\text { copies/mL }(n=513) \\
\text { AOR }(95 \% \mathrm{Cl}) ; p \text { value }\end{array}$ \\
\hline Mortality & - & - & & & \\
\hline Rural living & - & $2.13(1.07-4.27) ; 0.033$ & - & $0.66(0.46-0.93) ; 0.019$ & - \\
\hline Sex (male) & $\begin{array}{l}1.55(1.05-2.28) ; \\
0.026\end{array}$ & $2.36(1.28-4.37) ; 0.006$ & - & - & - \\
\hline $\begin{array}{l}\text { Age at study enrolment } \\
\text { ( } \geq 15 \text { years) }\end{array}$ & - & $0.32(0.16-0.62) ; 0.001$ & $0.82(0.62-1.10) ; 0.181$ & & \\
\hline Sexually infected & $\begin{array}{l}1.71(1.06-2.74) ; \\
0.028\end{array}$ & $0.28(0.15-0.52) ;<0.0001$ & - & - & - \\
\hline Decentralised care & & - & $1.39(1.06-1.83) ; 0.018$ & - & - \\
\hline Time on ART ( $\geq 2$ years) & & & $3.42(2.14-5.47) ;<0.0001$ & $1.72(1.09-2.72) ; 0.020$ & 1.70 (1.12-2.58); 0.013 \\
\hline $\begin{array}{l}\text { Age at most recent } \mathrm{VL} \\
(\geq 15 \text { years) }\end{array}$ & & & & $0.54(0.39-0.75) ;<0.0001$ & $0.72(0.55-0.94) ; 0.017$ \\
\hline
\end{tabular}

AOR Adjusted odds ratio, ART Antiretroviral therapy, Cl 95\% Confidence interval, VL Viral load

Note: Adjusted odds ratios are only indicated for variables remaining in the final model for each outcome. Empty boxes indicate variables that were not

applicable to analyses. Boxes filled with a dash indicate variables not included in the final model because they had failed to reach significance in prior models

evaluate both the timing and rate of viral suppression for a large cohort of adolescents across facilities in a sub-Saharan African public healthcare setting [4, 24].

By tracing participants in the community and across all 52 public facilities, this HIV care cascade adjusted for under-reported mortality and silent transfers in estimates of LTFU. At $16.9 \%$, this study suggests that the true rate of LTFU is higher than previously reported for vertically infected adolescents in sub-Saharan Africa. This difference may result from this study's inclusion of adolescents sexually infected with HIV, a sub-population previously found to be at higher risk for LTFU $[5,24]$.

This study found that roughly $38 \%$ of adolescents designated as LTFU by facilities were misclassified due to under-reported mortality and silent transfers. The importance of including silent transfers for accurately assessing patient outcomes has been noted elsewhere, particularly in settings like South Africa without universal unique patient identifiers [25]. The high rate of silent transfer may reflect adolescents' residential mobility; adolescents' actively self-specialising care services across multiple sites; or the effect of decentralising HIV care, through which patients' care is distributed across healthcare levels $[25,26]$. Therefore, facility-based approaches to HIV care and treatment in South Africa must account for adolescents' mobility across sites.

Findings suggest that South African adolescent progression to the final "90" of viral suppression in the UNAIDS 90-90-90 treatment goal remains low, at $47.5 \%$ for ART-initiated adolescents aged 10-19 years. Therefore, this study extends evidence on the adolescent HIV care cascade in South Africa, beyond ART initiation rates previously reported by Maskew and colleagues to the next step of viral suppression, linked across

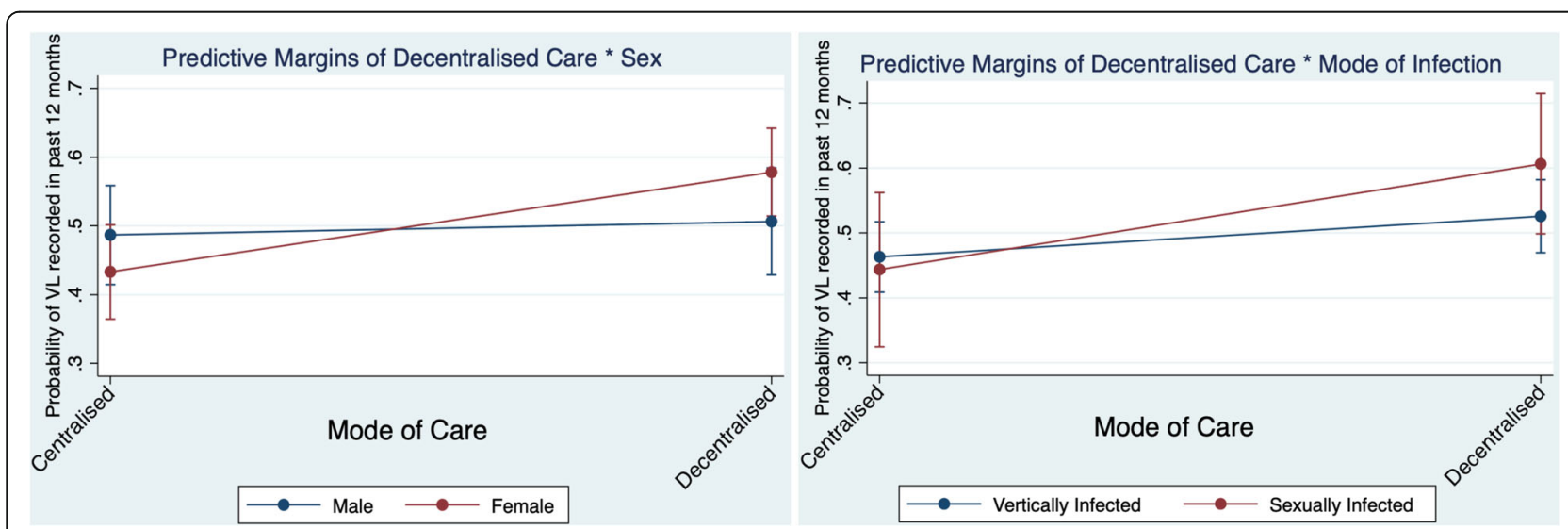

Fig. 2 Predicted probabilities of decentralised care by sex (left) and mode of infection (right) on recent viral load 
healthcare facilities [10]. This low rate of viral suppression in clinical records corroborates previously published findings on ART adherence from this adolescent cohort, which identified 49\% self-reported past-week ART adherence among adolescents at the study's 18-month follow-up interview [14]. Hence, findings suggest that critical challenges persist in adolescent attainment of consistent ART adherence and viral suppression after treatment initiation.

Further, this study investigated the critical implementation question of the frequency of viral load testing. This operational outcome reflects the extent to which adolescent HIV care in South Africa's decentralised health system actually adheres to national guidelines. National guidelines recommend at least annual viral load testing for all adolescents, even in the most decentralised community-based care models [11]. We found that only half of adolescents with available viral loads had their most recent viral load tested in the past 12 months. This rate of adolescents' viral load testing is far below the provincial estimate of $80 \%$ of all ART-initiated patients having a viral load within the past 12 months [27]. Although adolescents' viral load availability may be high, these results suggest that adolescents' viral loads are often out-dated. Because viral load monitoring is the primary approach for South African healthcare providers to determine patients' clinical care needs, the lack of recent viral load data available in routine clinical records may significantly reduce their ability to make appropriate care decisions [11].

Only $23.2 \%$ of all adolescents were documented to be virally suppressed in the past year. This low rate of pastyear viral suppression potentially provides a more meaningful representation of the current state of adolescent HIV care in South Africa. As younger adolescents age and become sexually active and have children, routine viral load monitoring and suppression become even more crucial for prevention of onwards transmission to partners and children [1]. While longer time on ART and receiving decentralised care were associated with having a viral load reported in the past 12 months, the protective effect of decentralised care was stronger for females and for sexually infected adolescents. These subpopulations may be more likely to engage with decentralised care through access to sexual and reproductive health services at primary care clinics.

This study has several limitations. Because study eligibility was limited to ART-initiated adolescents, findings are not generalisable to rates of viral suppression for adolescents living with HIV who have not been tested or initiated on ART, in the first two steps of the UNAIDS 90-90-90 target and care cascade. However, this study's inclusion of adolescents who were LTFU or disengaged from care minimised the risk of sample selection bias.
Because this retrospective cohort study analysed routine clinical records, it is only able to present estimates based on data that would be available to healthcare providers in facilities. Rates of viral suppression presented in this study represent the proportion of adolescents known and documented to have achieved viral suppression. Although adolescents without any electronic or paperbased viral load records could potentially be virally suppressed, this information was not available. In line with previous studies, these adolescents were presumed to be disengaged from care and thus likely to have unsuppressed viral loads $[6,28]$.

Additionally, the present study is unable to systematically identify precise reasons for out-dated or missing viral loads for each participant. Potential factors include both provider-related challenges (inconsistent recordkeeping systems resulting in missed scheduling, invalid test results, or lost test results), and patient-related challenges (true disengagement from care, being unwilling or unable to queue for blood tests, or having a proxy pick up medications and report on their health) [29, 30]. Potential transfers to facilities beyond the health district or into private care were not captured.

However, this study has several significant methodological strengths. Notably, participant tracing in the community allowed for correction of facilities' mortality records. Tracing individual patients across 52 healthcare facilities enabled a more accurate evaluation of adolescents' health by including unrecorded patient-initiated silent transfers to new facilities [24, 25]. This improved estimates for not only LTFU but also most recent viral loads. Analysis of clinical records through December 2017 also allowed for evaluation of adolescent viral load monitoring and HIV care outcomes in the era of universal test and treat. These outcomes reflect the current reality of how ART-initiated adolescents are receiving follow-up care and responding to treatment within a healthcare system responsible for a larger patient population than even before. Additionally, through linking adolescents' self-reported information to clinical records, this cohort study was able to evaluate the effects of individual-level predictors on progression along the HIV care cascade, which has not been possible in previous analyses of records from national databases [10, 24]. Finally, by evaluating adolescents' clinical records across 52 public facilities in the Eastern Cape, findings potentially represent outcomes for adolescents in similar settings across South Africa and sub-Saharan Africabeyond findings from highly resourced and externally supported clinics.

Further longitudinal analysis of outcomes is required to investigate the cyclical nature of engagement with HIV care, in order to identify when and which adolescents disengage from the HIV care cascade [31]. Future 
research within this adolescent cohort should also examine self-reported clinical care experiences as predictors of HIV care outcomes. Identifying reasons for gaps in the operational outcomes from this HIV care cascade and strategies to improve timely viral load monitoring for adolescents is critical for effective programmatic and policy changes.

\section{Conclusions}

This study documents progression of ART-initiated adolescents across the HIV care cascade to viral suppression in a sub-Saharan African public healthcare setting, accounting for mortality and silent transfers. Adolescents living with HIV in the Eastern Cape, South Africa, demonstrate low rates of viral suppression, and viral load monitoring practices fall well below national guidelines. The effectiveness of UNAIDS 90-90-90 goals for achieving HIV treatment success requires not only viral suppression but also routine, up-to-date viral load monitoring and recording.

\section{Supplementary Information}

The online version contains supplementary material available at https://doi. org/10.1186/s12879-020-05742-9.

Additional file $\mathbf{1}$ Table $\mathbf{S 1}$. Full results from sequential multivariable logistic regression analyses of associations between baseline sociodemographic and treatment-related variables and operational HIV care outcomes.

Additional file $\mathbf{2}$ Table S2. Full results from sequential multivariable logistic regression analyses of associations between baseline sociodemographic and treatment-related variables and viral load outcomes.

\section{Abbreviations \\ AOR: Adjusted odds ratio; ART: Antiretroviral therapy; ARV: Antiretroviral; $\mathrm{Cl}$ : Confidence interval; HIV: Human immunodeficiency virus; IQR: Interquartile range; LTFU: Loss to follow-up; NNRTI: Non-nucleoside reverse transcriptase inhibitor; NRTI: Nucleoside reverse transcriptase inhibitor; PI: Protease inhibitor; TB: Tuberculosis; UNAIDS: Joint United Nations Programme on HIV/AIDS; VL: Viral load; WHO: World Health Organization}

\section{Acknowledgements}

The authors thank the adolescents, healthcare facilities, and healthcare facility staff who participated in the Mzantsi Wakho study. We also thank the superb Mzantsi Wakho fieldwork teams as well as the University of Oxford and University of Cape Town support teams. We especially thank the Mzantsi Wakho study research assistants whose work was primarily clinic-based: Amanda Mbiko, Zoliswa Marikeni, and Pumza Bellem.

\section{Authors' contributions}

Study conception: RH, ET, LC; Study design: RH, ET, NB, LC; Data collection: $\mathrm{RH}, \mathrm{ET}, \mathrm{NB}$; Data analysis: RH, ET, LC; Manuscript drafting: RH, ET; Manuscript review and finalization: $\mathrm{RH}, \mathrm{ET}, \mathrm{NB}, \mathrm{LC}$. All authors have read and approved the manuscript.

\section{Funding}

The study is co-funded by the UK Medical Research Council (MRC) and the UK Department for International Development (DFID) under the MRC/DFID Concordat agreement, and by the Department of Health Social Care (DHSC) through its National Institutes of Health Research (NIHR) [MR/R022372/1], the Nuffield Foundation [CPF/41513], Janssen Pharmaceutica N.V., part of the Janssen Pharmaceutical Companies of Johnson \& Johnson, Evidence for HIV
Prevention in Southern Africa (EHPSA), a UKAID programme managed by Mott MacDonald [MM/EHPSA/UCT/05150014], the Regional Inter-Agency Task Team for Children Affected by AIDS - Eastern and Southern Africa (RIATTESA), UNICEF Eastern and Southern Africa Regional Office, Research England [0005218] and the International AIDS Society through the CIPHER grant (155Hod; 2018/625-TOS). This project was also funded by the UK Research and Innovation Global Challenges Research Fund award [ES/S008101/1]. Further support for $\mathrm{RH}$ is provided by the Clarendon Fund $(\mathrm{RH})$ and the Economic and Social Research Council [ES/J500112/1]. ET was also supported by the Clarendon-Green Templeton College Scholarship Fund (ET). Additional support for LC is provided by the European Research Council (ERC) under the European Union's Seventh Framework Programme [FP7/2007-2013/ERC grant agreement no 313421], the ERC under the European Union's Horizon 2020 research and innovation programme (grant agreement no 737476), and the Economic and Social Research Council [IAA-MT13-003]. It is made possible by the generous support of the American people through the United States Agency for International Development (USAID). Further support for the project is received from the John Fell Fund [161/033; 103/757], the Leverhulme Trust [PLP-2014-095] and the University of Oxford's ESRC Impact Acceleration Account [IAA-MT13-003/1602-KEA-189/K1311-KEA-004]. The views expressed are those of the authors and do not necessarily reflect the views of the aforementioned funders. The funders had no role in the design of the study, data collection and analysis, interpretation of findings, or manuscript preparation.

\section{Availability of data and materials}

The datasets generated and analyzed for the current study are not publicly available due to ethical and confidentiality constraints but can be available from the corresponding author on reasonable request within the purview of prior ethical approvals.

\section{Ethics approval and consent to participate}

Ethical approval to obtain and analyse raw data was given at four levels. At the university level, ethical approvals for the full study protocol were provided by the University of Oxford (SSD/CUREC2/12-21) and University of Cape Town (CSSR 2013/4; 2017/3). At the provincial government level, ethical approval was given by the Eastern Cape Department of Health and Department of Basic Education. At the healthcare facility level, approvals were obtained through the ethical review boards of each participating healthcare facility. Finally, ethical approval and consent to participate were obtained at the individual participant level, in line with protocols further described in this section. At both study waves, adolescent participants and their caregivers provided voluntary, informed, and written consent for participation, including interviews and access to adolescents' clinical records. Initial review of patient registers in healthcare facilities was only used to identify eligible participants, and further access to clinical records was obtained only after adolescents consented to study participation. In cases of low literacy among adolescents or caregivers, all information and consent procedures were read aloud in the participant's preferred language. There were no financial incentives for study participation, but all participants received a certificate of participation, snacks, and a small gift pack including pencils and soap. Adolescents who refused to participate were still given snacks.

\section{Consent for publication}

Not applicable.

\section{Competing interests}

The authors declare that they have no competing interests.

\section{Author details}

${ }^{1}$ Department of Social Policy and Intervention, University of Oxford, 32 Wellington Square, Oxford OX1 3DW, UK. ${ }^{2}$ Centre for Social Science Research, University of Cape Town, Cape Town, South Africa. ${ }^{3}$ Department of Sociology, University of Cape Town, Cape Town, South Africa. ${ }^{4}$ Department of Nursing Science, Faculty of Health Sciences, University of Fort Hare, Alice, South Africa. ${ }^{5}$ Department of Psychiatry and Mental Health, University of Cape Town, Cape Town, South Africa. 
Received: 12 February 2020 Accepted: 27 December 2020

Published online: 13 January 2021

\section{References}

1. UNAIDS. All In to \#EndAdolescentAIDS. Geneva, 2015

2. Ending UNAIDS. AIDS: Progress Towards the 90-90-90 Targets. In: Geneva; 2017.

3. Data UNAIDSAIDS. Geneva. In: 2017; 2016.

4. Slogrove AL, Sohn AH. The global epidemiology of adolescents living with HIV: Time for more granular data to improve adolescent health outcomes. Current Opinion in HIV \& AIDS. 2018;13(3):170-8.

5. Adejumo OA, Malee KM, Ryscavage P, Hunter SJ, Taiwo BO. Contemporary issues on the epidemiology and antiretroviral adherence of HIV-infected adolescents in sub-Saharan Africa: a narrative review. Journal of the International AIDS Society. 2015;18(1):20049.

6. Zanoni BC, Archary M, Buchan S, Katz IT, Haberer JE. Systematic review and meta-analysis of the adolescent HIV continuum of care in South Africa: the Cresting Wave. BMJ Global Health. 2016;1(3):e000004

7. MacCarthy S, Hoffmann M, Ferguson L, et al. The HIV care cascade: models, measures and moving forward. Journal of the International AIDS Society. 2015;18(1):19395.

8. UNAIDS. 90-90-90. An ambitious treatment target to help end the AIDS epidemic. In: Geneva; 2014

9. Jose S, Delpech V, Howarth A, et al. A continuum of HIV care describing mortality and loss to follow-up: a longitudinal cohort study. The Lancet HIV. 2018;5(6):e301-8.

10. Maskew M, Bor J, MacLeod W, Carmona S, Sherman GG, Fox MP. Adolescent HIV treatment in South Africa's national HIV programme: a retrospective cohort study. The Lancet HIV. 2019;6(11):e760-e8.

11. Southern African HIV Clinicians Society. Guidelines for adherence to antiretroviral therapy in adolescents and young adults. South Africa: Johannesburg; 2017.

12. Human Sciences Research Council. The Fifth South African National HIV Prevalence, Incidence, Behaviour and Communication Survey, 2017: HIV Impact Assessment Summary Report. Cape Town, South Africa, 2019.

13. Wong VJ, Murray KR, Phelps BR, Vermund SH, McCarraher DR. Adolescents, young people, and the 90-90-90 goals: a call to improve HIV testing and linkage to treatment. AIDS (London, Enland). 2017;31(Suppl 3):S191-S4.

14. Cluver LD, Toska E, Orkin FM, et al. Achieving equity in HIV-treatment outcomes: can social protection improve adolescent ART-adherence in South Africa? AIDS care. 2016:28(Suppl 2):73-82.

15. Massyn N, Padarath A, Peer N, Day C. District Health Barometer 2016/2017. Durban. In: South Africa: Health Systems Trust; 2017.

16. Cluver L, Pantelic M, Toska E, et al. STACKing the odds for adolescent survival: health service factors associated with full retention in care and adherence amongst adolescents living with HIV in South Africa. Journal of the International AIDS Society. 2018;21(9):e25176.

17. Hodes R, Cluver L, Toska E, Vale B. Pesky metrics: the challenges of measuring ART adherence among HIV-positive adolescents in South Africa. Critical Public Health. 2018:29(2):179-90.

18. World Health Organization. Consolidated Guidelines on the Use of Antiretroviral Drugs for Treating and Preventing HIV Infection. Recommendations for a Public Health Approach. In: Geneva; 2016.

19. Slogrove AL, Mahy M, Armstrong A, Davies MA. Living and dying to be counted: What we know about the epidemiology of the global adolescent HIV epidemic. Journal of the International AIDS Society. 2017:20:21520.

20. He E, Toska E, Cluver L, Sherr L. Determining mode of transmission amongst South African adolescents: A validation procedure using a communitytraced sample of HIV-infected adolescents in South Africa. In: 22nd International Workshop on HIV Observational Databases; 2018, vol. 22-24. Spain: March; Malága; 2018

21. Hosmer Jr DW, Lemeshow S, Sturdivant RX. Applied logistic regression: John Wiley \& Sons; 2013.

22. Hochberg Y. A sharper Bonferroni procedure for multiple tests of significance. Biometrika. 1988;75(4):800-2.

23. Buis ML. Stata tip 87: Interpretation of interactions in nonlinear models. Stata Journal. 2010;10(2):305-8.

24. Slogrove AL, Schomaker M, Davies M-A, et al. The epidemiology of adolescents living with perinatally acquired HIV: A cross-region global cohort analysis. PLoS medicine 2018; 15(3): e1002514.
25. Davies MA, Tsondai P, Tiffin N, et al. Where do HIV-infected adolescents go after transfer? Tracking transition/transfer of HIV-infected adolescents using linkage of cohort data to a health information system platform. Journal of the International AIDS Society. 2017:20(Suppl 3):21668.

26. South African National AIDS Council. Let Our Actions Count: South Africa's National Strategic Plan for HIV. In: TB and STIs, vol. 2017-2022. South Africa: Pretoria; 2017.

27. World Bank. Analysis of Big Data for better targeting of ART Adherence Strategies: Spatial clustering analysis of viral load suppression by South African province, district, sub-district and facility (April 2014-March 2015). In: Washington DC; 2016.

28. Long LC, Rosen SB, Brennan A, et al. Treatment outcomes and costs of providing antiretroviral therapy at a primary health clinic versus a hospitalbased HIV clinic in South Africa. PloS one. 2016;11(12):e0168118.

29. Euvrard J, Schulz T, Hilderbrand K, et al. How accurately do routinely reported HIV viral load suppression proportions reflect progress towards the 90-90-90 target in the population on antiretroviral treatment in Khayelitsha, South Africa? South African Medical Journal. 2019;109(3):174-7.

30. Granich R, Gupta S, Hall I, Aberle-Grasse J, Hader S, Mermin J. Status and methodology of publicly available national HIV care continua and 90-90-90 targets: A systematic review. PLoS medicine. 2017;14(4):e1002253.

31. UNAIDS. Prevention Gap Report. In: Geneva; 2016.

\section{Publisher's Note}

Springer Nature remains neutral with regard to jurisdictional claims in published maps and institutional affiliations.
Ready to submit your research? Choose BMC and benefit from:

- fast, convenient online submission

- thorough peer review by experienced researchers in your field

- rapid publication on acceptance

- support for research data, including large and complex data types

- gold Open Access which fosters wider collaboration and increased citations

- maximum visibility for your research: over $100 \mathrm{M}$ website views per year

At BMC, research is always in progress.

Learn more biomedcentral.com/submissions 